\title{
Probing Cosmic Rays in AGN and Clusters of Galaxies using radio observations
}

Nectaria A. B. Gizani*

Hellenic Open University, School of Natural Sciences and Technology, Tsamadou 13-15 and Ag. Andreou, 26222 Patra, Greece

E-mail: ngizani@eap.gr

\begin{abstract}
We search for radio structures not associated with Active Galactic Nuclei (e.g. haloes, mini halos, relics) in a sample of 70 Abell clusters for which we have radio, optical and X-ray data. Radio galaxies are known sources of high energy. Most galaxy clusters have cool cores with active galaxies at their centre. These AGN contain particle bubbles that show nonthermal radio emission. We are probing the role that intracluster magnetic fields, mergers, cooling flows, radio jets/shocks as well as radio halos/relics play in the formation and acceleration of cosmic rays.

Faraday rotation quantitatively establishes both cosmic ray and magnetic pressure component in the intracluster medium. Rotation measurements of background and cluster radio sources, together with X-ray observations provide the main evidence of large-scale intra-cluster magnetic fields and of densities of relativistic electrons. The intracluster medium of galaxy clusters is permeated by significant magnetic fields, which suggests that trapping and acceleration of cosmic rays by the external magnetic field could be occuring.

Radio spectral observations indicate the ageing of the emitting particles.

In the current paper we discuss results from the radio and X-ray analysis of two powerful radio galaxies from our sample namely Hercules A, and 3C 388.
\end{abstract}

ISKAF2010 Science Meeting

June 10 -14 2010

Assen, the Netherlands

\footnotetext{
* Speaker.
} 


\section{Introduction}

The intracluster medium (ICM) consists of relativistic particles (cosmic rays, CRs), weak $(\sim \mu \mathrm{G})$ magnetic fields and hot, $\mathrm{X}$-ray emitting gas. Therefore energy input from the ICM refers to gas heating, and injection of CRs and intracluster magnetic fields.

Features of diffuse low surface non-thermal radio emission of relativistic particles spiraling intracluster magnetic fields not directly associated with the galaxies of the cluster have been identified, and categorized as haloes, relics and minihalos. They are considered due to reacceleration of relic population of relativistic electrons, or proton proton collisions with the intracluster medium (ICM) (see Ferrari et al. 2008, and references therein).

In classical double radiogalaxies and quasars the relativistic (jets) are thought to force the radio lobes to extend supersonically in the warm gas. The Auger Collaboration correlates ultra high energy cosmic rays (UHECRs) with AGN. That is, UHECRs are fed by super-massive black holes at the centers of FR I and II radiogalaxies and can be accelerated by shocks, and turbulent plasma in the jets of AGN.

En $\beta$ lin et al., 1996 have concluded that radio galaxies are powerful enough to heat and support the cluster gas with injected cosmic-ray protons and magnetic field densities (permitted by Faraday rotation and gamma-ray observations of galaxy clusters) within a cluster radius of $\sim 1 \mathrm{Mpc}$.

Other energetic sources of cosmic rays both observationally and theoretically, apart from AGN (eg. [2]; [3]), can be shocks formed because of the jets continuously feeding the lobes with new material (eg. [2]; [12]), jets with particle composition of low energy $\mathrm{e}^{-} / \mathrm{e}^{+}$and relaticistic protons ([3]; [11]; [10]).

\section{Observations}

We search for radio structures not associated with the AGN (e.g. haloes, mini halos, relics) in a sample of 70 Abell clusters. The sample was selected upon the correlation of the radio power with the X-ray properties. We probe the role that cluster magnetic fields (via Faraday rotation and Inverse Compton Radiation arguments), merger events (through radio/X-ray interactions), cooling flow phenomena, jets, shocks, and nonthermal radio bubbles play in the formation, acceleration and propagation of cosmic rays. In the current paper we present the study of two powerful AGN from our sample. These radiogalaxies (RGs) are situated at the center of dense galaxy cluster environments called 'Hercules A' and '3C 388' -cluster named after their hosts. We assume $\mathrm{H}_{\circ}=$ $65 \mathrm{~km} \mathrm{~s}^{-1} \mathrm{Mpc}^{-1}$ and $\mathrm{q}_{\circ}=0$ throughout.

\section{Hercules A}

At the low redshift of $\mathrm{z}=0.154$ Hercules A ( $3 \mathrm{C} 348$, or Her A) is optically identified with a $\mathrm{cD}$ galaxy of a cluster, the dimmer and bigger of the two galaxies. HST data suggest possible kiloparsec rings of obscuration, aligned near the radio axis with a slight offset from the galaxy nucleus [1].

Hercules A is the fourth brightest radiogalaxy in the sky at low frequencies. It is classified as an FR1/2. There is a two-sided radio jet-morphology very different in appearance: Helical structures in the eastern jet and striking ring-like features in the western one. The Hercules A cluster is luminous in Xrays, although poor in optical galaxies. 
We have made VLA multiconfiguration, multifrequency total intensity and polarization observations at 1.4 arcsec resolution [6] together with ROASAT PSPC and HRI X-ray observations [7]. We have studied the environment of the AGN in terms of its magnetic field. EVN observations have also been taken to study Her A in pc-scales [8]. The jets and rings have dramatically flatter spectrum than the surrounding lobes and bridge, strongly suggesting that we are whitnessing a recently renewed outburst from the active nucleus. The lobes have steeper spectrum than found in typical radio sources, and steepens further towards the centre. We suggest that the dramatic asymmetry in the two sides of the radio emission is due to relativistic beaming and also because we are viewing the two lobes at different stages of the outburst. The compact core is optically thin and has a remarkably steep spectrum. It continues to be unresolved and very weak $(\sim 15 \mathrm{mJy})$ even at EVN (marcsec) resolution. The kpc-jets although collimated at short distances from the core within the lobes, they are misaligned in pc-scales with $\mathrm{a} \approx 35^{\circ}$ angle of misalignement in NW/SE direction, which may suggest a dense environment.

Our ROSAT X-ray data revealed a dense intracluster medium (ICM) typical for modest cooling flows. The PSPC spectrum shows a cool component of the ICM with $0.5 \leq \mathrm{KT}(\mathrm{keV}) \leq 1$ suggesting a multiphase gas when added to the BeppoSAX and ASCA results. The cluster emission is elongated parallel to the radio axis extending further than the radio lobes. We have found no obvious displacement of the ICM by the relativistic plasma, which would be apparent if depressions of the X-ray emission were coincident with the radio lobes. New Chandra observations have confirmed confinement of inner jets by the ICM and the presence of cavities, which unusually enough are not associated by the radio lobes [13].

We have estimated the external magnetic field by combining the X-ray with the radio results. The magnetic field in the ICM has a radial dependence of the form $\mathrm{B}(\mathrm{r}) \propto n(r)^{m-1}$, where $n(r)$ the electron density given by $n(r)=n_{\circ}\left[\left(r / r_{\circ}\right)^{\alpha_{1}}+\left(r / r_{\circ}\right)^{\alpha_{2}}\right] . \mathrm{n}_{e \circ}$ is the central value of the electron density and $\alpha_{1} \sim .7$ and $\alpha_{2} \sim 2.0$. The tangling scale size is found to be $4 \leq 1_{\circ}(\mathrm{kpc}) \leq 35$. Tables 1 and 2 summarize the X-ray bolometric luminosity of the cluster, the X-ray luminosity associated with the AGN, the radio power of the radiogalaxy at $178 \mathrm{MHz}$, the total X-ray extent $\mathrm{r}_{t}$ ot, parameters from the modified King model fitted to the gas distribution ( $\beta$, central electron density, temperature of the gas, core radius $r_{c}$ ), whether there is a cooling flow or not, the external magnetic field derived by rotation measure and inverse compton arguments, the central cosmic ray energy $\varepsilon_{C R}$ and the production rate of gamma rays $\mathrm{dn}_{\gamma} / \mathrm{dt}$.

\section{$3 \mathrm{C} 388$}

The optical galaxy $(z=0.0908)$ is one of the brightest cDs. It is a relatively small classical double FRII. Its faint halo in the eastern lobe with steep spectrum and high polarization, and the relic of an older jet activity are associated with the individual galaxy rather than the cluster as a whole. The western side presents a similar low surface radio emission. There is a luminous jet contained within the western lobe and a possible counter-jet.

3C 388 is at the centre of a poor cluster with very dense ICM. Our ROSAT HRI observations (energy range $0.1-2.4 \mathrm{keV}$ ) have found warm gas confining the lobes of this powerful radio source. Emission is detected to a radius $\simeq 5 \times$ larger than the radio lobes. Tables 1 and 2 list the cluster parameters for the $3 \mathrm{C} 388$ cluster as explained above.

We found marginal evidence for distortion of the gas by the radio lobes. Chandra data cleared this ambiguity [9]. The analysis of the new data gave $\beta=0.444 \pm 0.003$, central hydrogen density 
of $8.3 \cdot 10^{4} \mathrm{~m}^{-3}$, gas temperature $\mathrm{kT} \simeq 3.5 \mathrm{keV}$ (although varying slightly between the nucleus and lobes region) and $\mathrm{r}_{c}=15 \mathrm{kpc}$.

\section{Discussion}

Over the lifetime of galaxy clusters, mergers and AGN hosts should have accelerated and injected fields and energetic particles. Their combined energy should be comparable to the presentepoch thermal energy content of the central region. The field topology is expected to be complicated, with local variations of field strength and turbulence present. All these suggest an unstable and time-dependent configuration of fields, cosmic-rays and thermal gas. The presence of turbulent magnetic fields in the ICM of the galaxy clusters as well as merger activity most likely show up as radio halos, relics and can accelerate cosmic rays.

Both Her A and 3C 388 are cD galaxies and powerful AGN at the centre of cooling flow, dense, but poor clusters. A model by West 1994 suggests that $\mathrm{cD}$ galaxies and powerful radiosources are formed through highly anisotropic mergers. However there are for example no radio haloes, relics present which would be expected as a result of a merger event.

A front shock (cocoon) surrounding the radio source and cavities unusually not associated with the radio lobes have been revealed in the Her A cluster [13]. The latter could suggest that radiosource evolution cannot be determined entirely by the global properties of the hot gas. Cavities are also found in the $3 \mathrm{C} 388$ cluster coincident with the lobes as well as a possible sub-cluster either falling towards the host or already passed through [9]. However morphological differences between radio and X-rays could either suggest that the relativistic electrons are distributed homogeneously over the lobe, whereas the magnetic field is amplified towards the lobe rim region.

CRs are trapped in the magnetic field in our Galactic plane, which is frozen into the interstellar gas through its ionized component. It is only natural to hypothesize that similar trapping of CRs could occur by the IC magnetic field. We have estimated the thermal energy content of the two clusters by using a $\beta$-model for the cluster gas where the electron density is given by $\mathrm{n}_{e}(\mathrm{r})=$ $\left.\mathrm{n}_{e \circ} /\left(1+\left(r / r_{c}\right)^{2}\right)^{3 \beta / 2}\right)$. Results for the magnetic fields estimates are shown in Table 2. We mention here that intracluster magnetic fields from Faraday screen observations for the powerful host RGs of the Hydra A and Cygnus A clusters revealed values as high as $\sim 35 h_{65}^{1 / 2} \mu \mathrm{G}$. A nonthermal phase could be suggested via cluster core magnetic fields on the order of $10 \mu$ G. Such strengths are directly observed in clusters with central radio galaxy and cooling flow, probably typical of all clusters. The other phase consists of cosmic ray protons, that have cooling times equal to or larger than the Hubble-time as we discuss in what followes.

After correcting for Faraday rotation we have found that the projected magnetic field closely follows the edge of the lobes, the jets and the rings of Her A. The result suggests that the lobe interior should be dominated by particle pressure, whereas the magnetic pressure should dominate in the shell region defined by the lobe boundary. Our X-ray analysis ([7]; [11]) has pointed out that the gas thermal pressure is greater than the minimum pressure in the radio lobes by an order of magnitude for our best estimate. This implies confinement of the radio structure of the AGN by the ambient ICM rather than by shocks. There is little entrainment, so the energy supply of the lobes mostly comes from relativistic particles and magnetic fields. To retain equipartition our results would require $\mathrm{B} \approx 3 \mathrm{~B}_{m e}$, where $\mathrm{B}_{m e}$ is the magnetic field found using minimum energy. 
Table 1: Cluster Parameters (see text for explanation)

\begin{tabular}{|c|c|c|c|c|c|c|c|c|}
\hline Cluster & $\begin{array}{l}L_{X}^{\text {bol }} \\
10^{36} \mathrm{~W}\end{array}$ & $\begin{array}{l}L_{X}^{A G N} \\
10^{35} \mathrm{~W}\end{array}$ & $\begin{array}{l}P_{178 M H z}^{A G N} \\
10^{26} \mathrm{WHz}^{-1} \mathrm{sr}^{-1}\end{array}$ & $\beta$ & $\begin{array}{c}n_{e \circ} \\
10^{4} m^{-3}\end{array}$ & $\begin{array}{l}k T \\
k e V\end{array}$ & $\begin{array}{l}r_{c} \\
k p c\end{array}$ & $\begin{array}{l}r_{t o t} \\
k p c\end{array}$ \\
\hline Her A & 48 & 20 & 19 & $0.74 \pm .03$ & 1 & $0.5-1$ & 121 & 2200 \\
\hline $3 C 388$ & 5 & 5 & .48 & $0.53 \pm .04$ & 1.5 & 3 & 33 & 180 \\
\hline
\end{tabular}

Table 2: Cluster Parameters-continued

\begin{tabular}{cccccc}
\hline $\begin{array}{c}\text { Cluster cooling } \\
\text { flow }\end{array}$ & $B_{R M}$ & $B_{I C}$ & $\varepsilon_{C R}$ & $d n_{\gamma} / d t$ \\
\hline Her $A$ & $y$ & $3-9$ & 4.3 & $2.4-4.8$ & $.94-1.9$ \\
$3 C 388$ & $\sim y$ & - & 3.8 & 21.6 & 12.6 \\
\hline
\end{tabular}

However as discussed by Leahy \& Gizani 2001 , the magnetic field should be below equipartition and therefore unimportant in the lobe dynamics. Hence "invisible" particles (relativistic protons, low energy $\mathrm{e}^{-} / \mathrm{e}^{+}$) should dominate. This is also confirmed by Ensslin et al. 1996 who suggested the production of very energetic relativistic protons, besides relativistic electrons, in the lobes of radio galaxies to explain cosmic ray energies $>3 \cdot 10^{18} \mathrm{eV}$ reaching Earth.

Adopting the the fitting results of the correlation between the RGs' jet power vs luminosity at $2.7 \mathrm{GHz}$ by $\mathrm{En} \beta$ lin et al. 1996 , the energy input into the central region of clusters from the host RG is $\simeq 0.7 \cdot 10^{22} \mathrm{Wkpc}^{-3} h_{65}^{-2}$ for the power law $\mathrm{b}=0.82$. The injected jet power may dissipate and heat the gas, or could accumulate and support the ICM (magnetic fields and particles). Following the formalism by Ensslin et al. 1996 we estimate the central cosmic ray energy $\varepsilon_{C R(r)}$ from the thermal energy density as $\varepsilon_{C R(r)}=3 n_{\circ e}(r) \cdot k T \cdot \alpha_{C R}$, assuming the scaling ratio between the thermal and $\mathrm{CR}$ energy densities to be $\alpha_{C R} \simeq 1$. The production rate for gamma rays above $100 \mathrm{MeV}$ by $\pi_{\circ^{-}}$ decay after hadronic interactions of the energetic protons with the background gas is estimated from $d n_{\gamma}(>100 \mathrm{MeV}) / d t=3 q_{\gamma} n_{e \circ}^{2} k T \alpha_{C R}$. The parameter $q_{\gamma}=0.39 \cdot 10^{-12} \mathrm{~m}^{3} \mathrm{~W}^{-1} \mathrm{~s}^{-2}$ applies to a proton spectrum similar in slope to that observed in the Galaxy. See Table 2 for the results.

The radiative cooling time (in Gyr) in the central regions of the Her A cluster is $\simeq 6$ Gyrs for the hot phase and 2 Gyrs for the cool phase. For the 3C 388 cluster is 3.7 Gyrs. Comparison of these values with the age of the universe ( $\sim 10 \mathrm{Gyrs})$ implies the existence of a cooling flow [14] suggested by the X-ray data. Synchrotron cooling time is too short for the relativistic electrons to diffuse in the whole lobes considering the growth speed of the lobes [15]. While the radio emitting medium leaves the lobes diffusing into the cluster medium, the CR part of the outflow is dissipated. Relativistic electrons loose their energy via numerous cooling mechanisms: relatively quickly through synchrotron emission whilst spiraling the cluster magnetic fields ; also through Compton scattering through collisions with photons of the cosmic microwave background. These 
processes are not effective for energetic protons whose Compton and synchrotron cooling times are much longer than the Hubble-time. A possible mechanism for slowing down energetic protons is suggested via electronic excitations (see Ensslin et al. 1996 and references therein).

We note that both cooling flow clusters have extended radio emission. The short cooling time of the emitting cosmic ray electrons and the large extent of the radio sources suggest an ongoing acceleration mechanism in ICM. The cooling of the electrons is visible in the steepening of the spectral index of radio emission close to radio galaxies in clusters. Steep spectral indices found in the lobes of Hercules A and 3C 388 imply short lifetimes of radiating particles and also to some extent re-acceleration of the electrons suggesting energy redistribution in the ICM. If cooling is significant to the Hubble time then the cooling radius of the Her A cluster is $\sim 90 \mathrm{kpc}$ less than the core radius of $\sim 120 \mathrm{kpc}$ found by our X-ray analysis. The energy lost by the energetic particles could be gained by the magnetic fields also heating the ICM. Clusters seem to grow. As a result the energy of CRs in the ICM should increase adiabatically. Additional CR sources such as supernovae, shocks, in-situ acceleration could also compensate for the energy loss.

In the current work we confirm the presence of extragalactic magnetic fields in the ICM which can accelerate cosmic rays. There are no radio features associated with the studied clusters but depressions in the X-ray emission coincident with the radio lobes. We have found that the jets of the host AGN should contain low energy $\mathrm{e}^{-}, \mathrm{e}^{+}$, and relativistic $\mathrm{p}$ and are capable of feeding cosmic rays.

\section{References}

[1] Baum, S.A., O’Dea, C.P., de Koff, S., Sparks, W., Hayes, J. E., Livio, M. \& Golombek, D. 1996, ApJL, 465, 5

[2] Dremmer, C.D., Razzaque, S., Finke, J.D. \& Atoyan A. 2009, New J. Phys., 11, 065016

[3] En $\beta$ lin, T. 2004, J Kor Astron Soc, 37, 1

[4] En $\beta$ lin, Torsten A., Biermann, Peter L., Kronberg, Philipp P. \& Wu, Xiang-Ping 1997, ApJ, 477, 560

[5] Ferrari C., Govoni F., Schindler S., Bykov A.M. \& Rephaeli Y. 1992, Clusters of galaxies: beyond the thermal view, in Space Science Reviews, ed. J.S. Kaastra, [astro-ph/0801.0985v1]

[6] Gizani, Nectaria A.B. \& Leahy, J. P. 2003, MNRAS, 342, 399

[7] Gizani, Nectaria A.B. \& Leahy, J. P. 2004, MNRAS, 350, 865

[8] Gizani, Nectaria A.B., Garrett, M. A. \& Leahy, J. P. 2002, PASA, 19, 1

[9] Kraft, R.P., Azcona, J., Forman, W.R., Hardcastle, M.J., Jones, C., \& Murray 2006, ApJ, 639, 753

[10] Leahy, J. P. \& Gizani, Nectaria A.B. 2002, NewAR, 46, 117

[11] Leahy, J. P. \& Gizani, Nectaria A.B. 2001, ApJ, 555, 709

[12] Niemec, C.D. \& Ostrowski, M. 2006, ApJ, 641, 984

[13] Nulsen, P.E.J., Hambrick, D.C., McNamara, B.R., Rafferty, D., Birzan, L., Wise, M.W., \& David, L.P. 2010, ApJL, 625, No 1, 9

[14] Sarazin, G. L. 1986, Phys. Rev., 58, 1

[15] Scheuer, P.A.G. 1995, MNRAS, 277, 331

[16] West, M. J. 1994, MNRAS, 268, 79 